\title{
INTERCOMPARISON AMONG PLASMA WAKE MODELS FOR PLASMASPHERIC AND IONOSPHERIC CONDITIONS
}

\author{
U. SAMIR \\ Department of Geophysics and Planetary Science, Tel-Aviv University, Ramat-Aviv, Israel and \\ Space Physics Rescarch Laboratory, The University of Michigan, Ann Arbor, MI 48109, U.S.A. \\ R. H. COMFORT and K. H. WRIGHT, JR. \\ Department of Physics, The University of Alabama in Huntsville, Huntsville, AL 35899, U.S.A. \\ and \\ N. H. STONE \\ Space Sciences Laboratory, NASA/Marshall Space Flight Center, Huntsville, AL 35812, U.S.A.
}

(Received in final form 18 June 1987)

\begin{abstract}
Intercomparisons are made among the angular distributions of ions in the wake of a body moving through a space plasma as computed from three different expressions (models). Both subsonic and supersonic relative flows are considered in order to examine the wake current depletion ratios under conditions realistic for the topside ionosphere and plasmasphere. Results of these comparisons demonstrate the importance of including the thermal flux at low Mach numbers and of taking into account the angular acceptance of ion detectors in making theory-experiment comparisons. Gradients in the angular variations of the fluxes are found to be steeper near the wake-ambient interface than closer to the maximum rarefaction region of all models, although quantitatively there is considerable variation among the models. From examining the variations of the wake depletion ratio parametrically with Mach number and normalized potential over ranges characteristic of the plasmasphere and topside ionosphere, we find considerable variation with both parameters, with sensitivity to normalized potential increasing dramatically with Mach number. Overall, however, the Mach number variation appears to be the more significant over this range of parameters.
\end{abstract}

\section{INTRODUCTION}

Some aspects of the electrodynamic interaction between a small scientific satellite and the terrestrial plasmasphere were discussed recently in Samir et al. (1986). This paper is designated hereafter as paper I. The parametric results discussed in paper I, based on in situ measurements from the Retarding Ion Mass Spectrometer (RIMS) on Dynamics Explorer 1 $(D E-1)$, show the variations of $\alpha$

$$
\left(\equiv \frac{I_{+}(\text {wake })}{I_{+}(\text {ram })}-\frac{I_{+}\left(165^{\circ}<\theta<180^{\circ}\right)}{I_{+}\left(\theta=0^{\circ} \pm 15^{\circ}\right)}\right)
$$

with $R_{\mathrm{D}_{1}}\left(\equiv R_{0} / \lambda_{\mathrm{DI}_{1}}\right), S$ (Average), $S$ (Specific), and with $M_{+}$(Average), where $R_{0}=$ effective radius of the satellite, $\lambda_{\mathrm{Di}}=$ ion ambient Debye length, $S$ (average $/$ specific) $=$ average $/$ specific ionic Mach number, $M_{+}$(average) $=$average ion mass, and $\theta=$ angle of attack of the detector axis. In the above study no quantitative conclusions were obtained regarding the variation of

$$
\alpha=f\left(\phi_{\mathrm{N}} \equiv \frac{e \phi_{\mathrm{s}}}{K T}\right)
$$

where $\phi_{\mathrm{s}}=$ satellite potential (with respect to the ambient plasmaspheric plasma) and $T=$ ion temperature. Furthermore, it was shown in paper I that a "neutral approximation", namely, an approximation in which the motion of the ions is treated as if they were "neutral" particles, applied reasonably well to subsonic/transonic flows corresponding to plasmaspheric conditions. Based on earlier studies, e.g. Samir et al. (1973), Samir and Willmore (1965) and Henderson and Samir (1967), for supersonic/ hypersonic flows, corresponding to ionospheric conditions, it can be stated that the "neutral" approximation better describes the situation for a subsonic/transonic flow regime than it does for a supersonic/hypersonic flow regime.

The present paper extends the results of paper I, in the sense that it further explores properties of "neutral approximation" models and another model and provides information regarding the relative significance 
of the ionic Mach number and body potential in determining the ion distribution in the wake. More specifcally, we: (1) compare calculated results of

$$
\alpha=\frac{I_{+}(\theta)}{I_{+}(\text {ram })}
$$

for several $\Delta \theta$ ranges as obtained by three theoretical models, two of which utilize the "neutral approximation", while the other employs a Debye sheath model; (2) use the Debye sheath model to assess the relative significance of the parameter $\phi_{\mathrm{N}}$ vs the parameter $S$ in determining $\alpha$ for three angular $(\theta)$ ranges within the satellite wake; and (3) use the Debye sheath model to examine the effect of a limited angular response, as compared with a full hemispheric response, so that geometric and physical effects can be separated. This study assumes a single ion species plasma, the properties of which are important only in their effect on the dimensionless parameters employed in the study.

In this paper we do not discuss in detail the various aspects of the "body-plasma" interaction to space plasma physics at large. This was done in some detail in paper I, for plasmaspheric plasmas, and in Al'pert (1983) for a variety of space plasmas. However, a few general points regarding the specific interaction of a satellite with its environmental space plasma are appropriate. First, it should be noted that such an interaction can serve as a model for a much wider range of interactions directly relevant to solar system plasmas. Examples are the interactions between Io, Ganymede/Titan with the magnetospheres of their parent planets Jupiter/Saturn, and the interaction of the moon, Venus, Mars and comets (i.e. non-magnetized bodies) with the solar wind. Second, investigating "body"-plasma interactions under wide ranges of plasma and body parameters will lead eventually to a unified approach in dealing with the overall problem of body-plasma interactions.

In addition to the above-mentioned science aspects, there is also a practical aspect, namely, the application of satellite-plasma interaction results to the correct interpretation of low energy particle and field measurements made on board spacecraft. This should be particularly so for measurements to be conducted via the space shuttle and in the future via the space station. And last, but not least, is the contribution "body-plasma" interaction studies may have for the optimal planning, design and location of probes in future space missions.

In the present study we are concerned with the wake region only. In this region plasma waves are excited, rarefaction waves (or shocks) propagate, instabilities are generated, wave-particle interactions take place and turbulent regions as well as potential wells exist. In principle, such phenomena are to be expected, since in the wake region, plasma streams collide, ion fronts propagate, ions are accelerated and strong density gradients exist at the body-plasma interface (Stone, 1981a). It now becomes clear that part of the structural wake characteristics can be interpreted in terms of processes involved in the expansion of a rarefied plasma into a vacuum (e.g. Samir et al., 1983; Wright et al., 1985, 1986; Singh and Schunk, 1982; Gurevich et al., 1973; Gurevich and Meshcherkin, 1981a,b).

\section{PRESENTATION OF THE MODELS}

Three models (Gurevich et al., 1969; Samir and Willmore, 1965; Whipple et al., 1974) are used to compute the angular distribution of ion fluxes around a conducting body moving through a rarefied plasma. It should be noted that these models, frequently used in various body-plasma interaction studies, do not reflect the large variety of physical processes (noted above) which are believed to take place in the wake region (e.g. Stone, 1981a,b; Al'pert, 1983; Samir et al., 1983; Gurevich et al., 1969; Raitt et al., 1984; Siskind et al., 1984; Murphy et al., 1986). However, such models are often used as part of complicated spacecraft charging computer codes (e.g. Katz et al., 1984) for theory-experiment comparisons in wake studies (e.g. Al'pert, 1983; Samir et al., 1986, 1973), when qualitative or semi-quantitative information is sought.

At the present time there is no agreed-upon theoretical model which describes the temporal and spatial distribution of charged particles and fields around a spacecraft under realistic conditions, particularly not for the wake region. This is true even for the elaborate numerical model of Parker (e.g. Parker, 1977, 1983; Samir and Fontheim, 1981). Before the inclusion of all of the complicated phenomena noted above in a wake model is attempted, it is important first to treat correctly the problem of ion flux vs angle-of-attack to a surface mounted detector. Since zero-order approximations (i.e. simplified models) are frequently used in comparisons between theory and experiments, it is worthwhile to make intercomparisons between the predictions of several such models. Accordingly, we have selected three models for comparison. The first two models used in this study (Gurevich et al., 1969, 1973 ; Samir and Willmore, 1965) are essentially "neutral approximation" expressions. These expressions are based on the assumption that ions can be treated as neutral particles, which implies that the effect of electric fields on particle trajectories can be ignored. 
They differ primarily in their treatment of the thermal flux component. The third model is a Debye sheath approximation. This model takes into account effects of the spacecraft potential, allowing us to examine its significance. This model also readily allows for a limited angular response, whereas the two neutral approximations assume full hemispheric angular acceptance.

The first model was discussed in detail by Gurevich et al. $(1969,1973)$, Gurevich and Pitaevsky $(1975)$ and by Al'pert (1983). In that model, the flux or current around a body, normalized to the flux or current in the ram direction, was given. The expression used here [equation (1)] is a single-ion modified version of an approximate expression [equation (98)] given in Gurevich et al. (1969). In the original expression (Gurevich et al., 1969), an angle $\theta_{0}$, which characterizes the position of the boundary of the region of maximum rarefaction, was included. This boundary characteristic is taken here to be represented by $\cos \theta_{0}=\cos 45^{\circ}=0.7071$. The angle $\theta_{0}=45^{\circ}$ is indeed an approximation but is reasonably sufficient for the purpose of the present study. This consideration is valid for spacecraft negative potentials which are not too large (i.c. for $\left|\phi_{\mathrm{s}}\right|<k T e / e^{\cdot} R_{\mathrm{D}}^{4 / 3}$ ). This approximation was used in the past in theoryexperiment comparisons using Explorer 31 data (Gurevich et al., 1969) for situations where $\left[\mathrm{H}^{+}\right]$was the major ionic constituent of the plasma. As used in this study, this expression is given by:

$$
\begin{aligned}
\alpha_{\mathrm{O}}=\frac{\left[1+\operatorname{erf}\left(S \cdot \cos \theta \cdot \cos \phi_{0}\right)\right]}{\left[1+\operatorname{erf}\left(S \cdot \cos \phi_{0}\right)\right]} \\
=\frac{[1+\operatorname{erf}(0.7071 \cdot S \cdot \cos \theta)]}{[1+\operatorname{erf}(0.7071 \cdot S)]}
\end{aligned}
$$

(note: $\theta=180^{\circ}$ for the maximum rarefaction zone in the wake axis, and $\theta=0^{\circ}$ for the ram direction). This expression is the one (of the three models used in the present study) most used in previous wake studies (see Al'pert, 1983 for detailed discussions).

The second model is based on the same reasoning as equation (1) in the paper of Samir and Willmore (1965), which is a modification of Whipple (1959) to include variation with ram angle. In the model as used in this paper, the normalized flux is given by

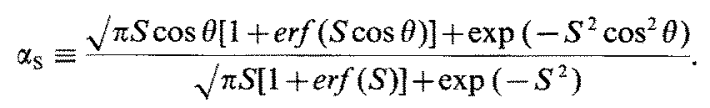

This will be referenced as the "Samir model". It will be noted that models (1) and (2) are similar except for the inclusion of an exponential term in the Samir model. This term represents the thermal flux, while the other terms give the ram flux associated with the body motion. Thus, comparison of these two models indicates the importance of including the thermal component of ion flux for the given conditions. The significance of the ion thermal motion was discussed in detail in Samir and Widjaja (1980), using two elaborate numerical models. This model would appear to be more appropriate than the "Gurevich" model for low Mach number flows.

The third model used in this study is based on equation (19) from Whipple et al. (1974). It assumes a spherically symmetric Debye sheath and a circular aperture with a specified half-angle acceptance cone. The current to a detector is formulated as the following integral

$$
\begin{aligned}
& J(\theta)=\exp \left(-\phi_{\mathrm{N}}-S^{2}\right) \\
& \cdot \iint \exp \left[-X-Z+2 S\left(X+Z+\phi_{\mathrm{N}}\right)^{1 / 2} \cos \theta \cos \alpha_{\infty}\right] \\
& \quad \cdot I_{0}\left[2 S\left(X+Z+\phi_{\mathrm{N}}\right)^{1 / 2} \sin \theta \sin \alpha_{\infty}\right] \mathrm{d} X \mathrm{~d} Z
\end{aligned}
$$

where:

$I_{0}$ is the modified Bessel function of order zero, $X$ is the initial transverse (relative to a detector aperture) kinetic energy normalized to $K T$, $Z$ is the corresponding initial normal kinetic energy, and

$\alpha_{\infty}$ is a particle direction at infinity relative to the detector normal,

$\phi_{\mathrm{N}}$ is the satellite potential normalized to $K T$

and the angular acceptance is incorporated into the integration boundaries.

This will be referenced as the "Whipple model". In order to use it, the integral is evaluated numerically in a thin sheath approximation, which has been found to be generally applicable to the plasmasphere (Comfort et al., 1982, 1985). In particular, it was noted by Comfort et al. (1985) that when spacecraft potentials were most negative $(-2$ to $-4 \mathrm{~V})$, the spacecraft was deep in the plasmasphere where the Debye length was small $\left(R_{\mathrm{D}_{1}} \leqslant 0.1\right)$. Largest positive potentials occurred in the outer plasmasphere where Debye lengths were large; but the numerical results are relatively insensitive to sheath thickness for positive potentials.

The depletion ratio $\alpha_{w}$ is given by the ratio of this integral evaluated for the angle of attack of interest to the integral evaluated in the ram direction $\left(\theta=0^{\circ}\right)$. For purposes of comparison, $\alpha_{\mathrm{w}}$ is evaluated for two aperture geometries. For the first, designated Whipple $\left(30^{\circ}\right)$, integration boundaries for the $D E-1 /$ RIMS aperture $\left(30^{\circ}\right.$ half-angular acceptance) were em- 
ployed, as discussed by Comfort et al. (1985). For the second, designated Whipple $\left(90^{\circ}\right)$, a full hemispheric aperture acceptance $\left(90^{\circ}\right.$ half-angle) is used so that direct comparisons can be made with the "neutral" approximation models without confusing geometric and physical effects. Comparisons between the results for the two aperture geometries then indicate the effects which geometry alone can cause.

The analytical formulation of the thin sheath limit in the $\operatorname{ram}\left(\theta=0^{\circ}\right)$ direction was shown by Comfort et al. (1982) to reduce to the neutral approximation of Whipple (1959) in the limit of a full hemisphere aperture acceptance angle. For other directions, when $\theta_{\mathrm{N}}=0$, the primary difference between the "Whipple" (90 aperture) and "Samir" models is due to the integrations over the angle variables in the "Whipple" model, which results in some differences to be seen below. The "neutral" approximation models are evaluated directly from their analytical expressions.

\section{RESULTS AND DISCUSSION}

\subsection{Intercomparisons among models}

Figure 1 shows variations of ion current ratios

$$
\alpha=\frac{I_{+}(\theta)}{I_{+}(\mathrm{ram})}
$$

with angle of attack $(\theta)$ for different ionic Mach numbers $(S)$, as obtained from the Whipple (W), Samir $(S)$ and Gurevich (G) models [see expressions (3), (2), (1), respectively] for the condition $\phi_{\mathrm{N}}=0$ (i.e. $\phi_{\mathrm{s}}=0$ ). The values for the ion currents represent averages over angle ranges $\pm 15^{\circ}$ about the $\theta$ direction. This is an effort to simulate conditions typically occurring for a spinning satellite. These averages are computed by evaluating the models at $5^{\circ}$ intervals and averaging over the specified range for both the ram and $\theta$ directions. It should be noted that for the $S=4.0$ case, double precision was required in evaluating the error function for the maximum wake region. Otherwise, values tended to "saturate" near values associated with minimum computable numbers rather than continuing to decrease as would be expected. In Fig. Ia, corresponding to Mach numbers $0.5,1.0$ and 2.0, only the Whipple $\left(30^{\circ}\right)$ case is shown. For $S=0.5$ and 1.0 , the Whipple $\left(90^{\circ}\right)$ curves differ from the Samir curves by about $2 \%$ or less at all angles, with maximum deviations occurring near $\theta=90^{\circ}$. For $S=2.0$ the differences between Samir and Whipple $\left(90^{\circ}\right)$ varied from about $30 \%$ near $\theta=90^{\circ}$ to less than $\frac{1}{2} \%$ at $\theta=180^{\circ}$, still small relative to the scale of variation of the other curves. For $S=4.0$, in Fig. $1 \mathrm{~b}$, the differences between the Whipple $\left(90^{\circ}\right)$ and Samir models are shown, since they are substantial enough to be clearly discriminated. Interestingly, even for this case, the differences at $\theta=180^{\circ}$ are only about $20 \%$, the largest differences (more than an order of magnitude) still occurring near $\theta=90^{\circ}$.

From Fig. 1, it is immediately evident that:

(i) At all Mach numbers, the differences between the Whipple $\left(30^{\circ}\right)$ and the Gurevich expressions arc the largest, with the Gurevich model consistently giving the highest values, the Whipple $\left(30^{\circ}\right)$ model the lowest, and the Samir land Whipple $\left.\left(90^{\circ}\right)\right]$ model giving intermediate values.

(ii) For any given angular range the differences between the models increase with increasing $S$; in particular, the differences are larger for typical ionospheric plasmas $(S \geqslant 2-4)$ than they are for typical plasmaspheric plasmas $(S \sim 0.5-2)$.

(iii) The differences between the Whipple (both) and the Samir models decrease as we proceed toward the maximum rarefaction zone $\left(\theta=180^{\circ}\right)$ in the wake.

(iv) The differences between the Gurevich and the Samir and Whipple models increase toward the maximum rarefaction zone in the wake.

In paper I, in situ measurements from the RIMS probe on board the Dynamics Explorer 1 satellite were compared with (essentially) the Samir expression. Specifically, $\left[\alpha(180)_{\text {theory }}\right]$ was compared with $\left[\alpha(180)_{\text {measurement }}\right]$ in the $S(A V)$ range of $0.4-1.2$ (i.e. subsonic/transonic flow), and it was found that

$$
\frac{\alpha(180)_{\text {theory }}}{\alpha(180)_{\text {experiment }}} \simeq 2-3 \text {. }
$$

Such a discrcpancy was not considered (by the authors of the paper) to be too serious considering the orders of magnitude discrepancies that we know exist in theory-experiment comparisons for the middle ionosphere (Samir et al., 1973; Samir and Fontheim, 1981). The latter comment is valid even for elaborate numerical models like that of Parker (Parker, 1983; Samir and Fontheim, 1981). Although the purpose of this paper is not to compare theory with experiment, the results from Fig. 1 above can be used for a qualitative assessment of the degree of theory/experiment agreement for the Gurevich expression.

Comparing the results for Gurevich with those of Samir, over the range $0.5 \leqslant S \leqslant 1.0$, we see that the Gurevich results are larger than the Samir results by factors of $\sim 3-7$. Hence, in a comparison with the above $D E$ experimental data the Gurevich model would provide a somewhat worse agreement compared with that of Samir. Earlier theory-experiment 


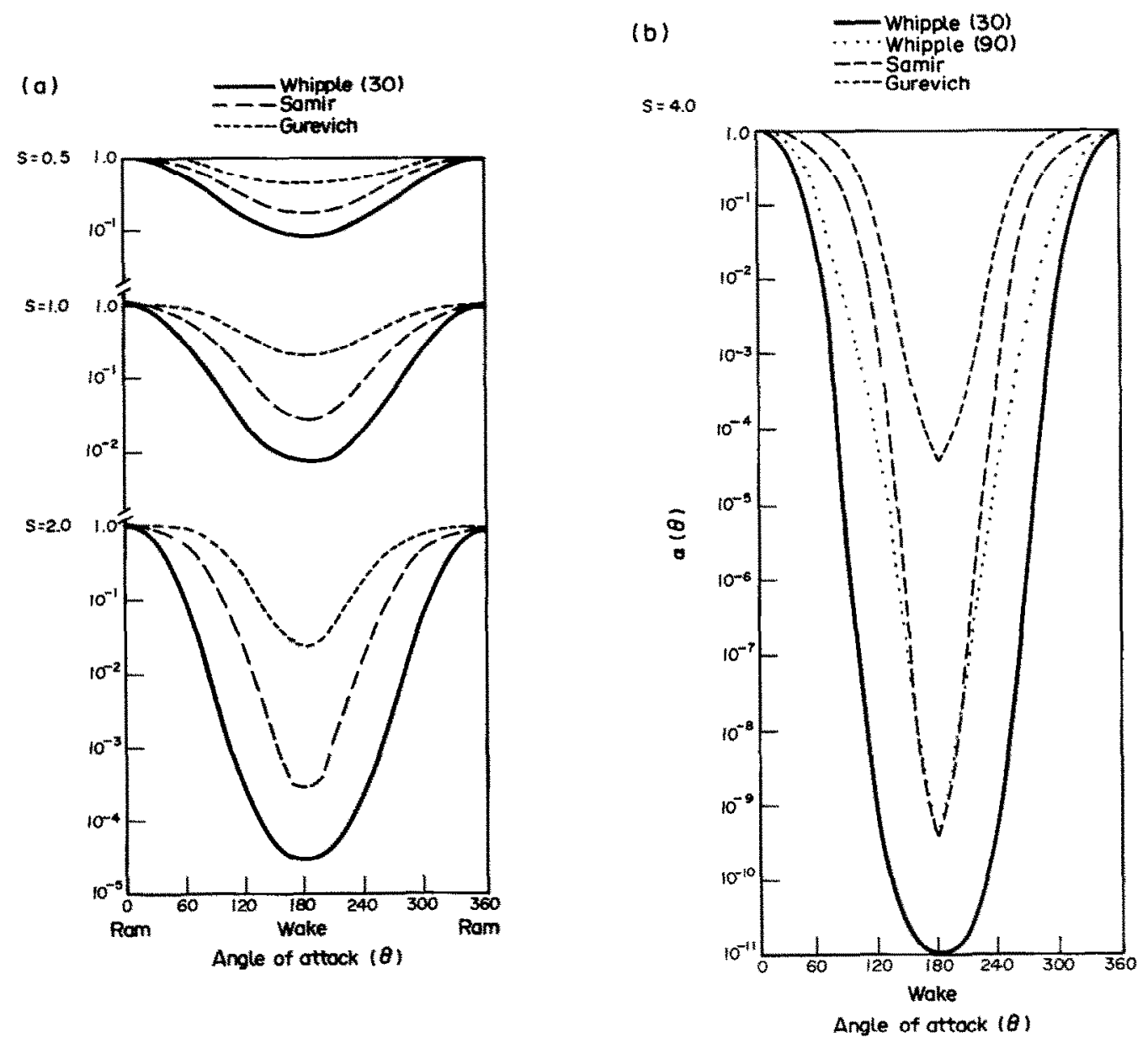

Fig. 1. VARIATION OF THE FLUX DEPLETION RATIOS $\alpha$ WITH ANGLE OF ATTACK $\theta$, WITH MACH NUMBER $S$ as A PARAMETER AND $\phi_{\mathrm{N}}=0$ FOR THE GUREVYCH, SAMIR AND WhPPLE MODELS.

(a) $S=0.5,1.0$ and 2.0 . The Whipple $\left(90^{\circ}\right)$ model has almost the same values as the Samir model. (b) $S=4.0$ with both Whipple $\left(90^{\circ}\right)$ and Whipple $\left(30^{\circ}\right)$ results displayed.

investigations in the ionosphere (i.e. in a supersonic/hypersonic flow regime) have shown that for specific cases the Gurevich expression yields results in better agreement with observations than results from the Samir expression (Gurevich et al., 1969 ; Samir et al., 1973). Results from these comparisons for the two different plasma flow regimes support the expectation that inclusion of thermal fluxes becomes increasingly important with decreasing Mach number. However, both neutral approximation expressions predicted ion fluxes in the wake which are much smaller than those observed.

If we make similar comparisons between the Samir and Whipple models, the Whipple $\left(30^{\circ}\right)$ results are factors of 2-3 lower than those of Samir over the range $0.5 \leqslant S \leqslant 1.0$. This would suggest the Whipple $\left(30^{\circ}\right)$ model might compare more favorably with the observations. Since the aperture geometry for this case corresponds to the $D E-1 /$ RIMS equivalent aperture, an improvement should be expected to occur for this model. However, in Fig. 1, the Whipple model is evaluated for $\phi_{\mathrm{N}}=0$, whereas the observations (paper 1) vary over the range $-3.6 \leqslant \phi_{N} \leqslant+0.9$. As we will see below, there are significant variations over this range of $\phi_{\mathrm{N}}$ which might be sufficient to give a different outcome in a detailed comparison. Similarly, for ionospheric comparisons, Fig. 1 would suggest that the Whipple model for any aperture half-angle significantly smaller than $90^{\circ}$ would underestimate the wake flux even more than the neutral models. However, the relatively large negative values of $\phi_{\mathrm{N}}$ characteristic of ionospheric satellites could sig- 
nificantly increase the Whipple model prediction of wake flux (see below).

Geometric effects can be seen by comparing the Whipple $\left(30^{\circ}\right)$ model with the Samir model in Fig. 1a or with the Whipple $\left(90^{\circ}\right)$ model in Fig. 1b. Because the plotted $\alpha$-values represent wake-to-ram current ratios, it is not immediately obvious that the smaller aperture acceptance should display the smaller ratio in the wake region. However, it does become clear when we consider the physical situation. The $90^{\circ}$ aperture averages over a larger angular range in all directions. When the detector points in the $\operatorname{ram}\left(\theta=0^{\circ}\right)$ direction, most of the current comes from the region near the detector axis; whereas when the detector points in the maximum wake direction $\left(\theta=180^{\circ}\right)$, most of the current comes from the outer limits of the angular acceptance. The comparison of the two detector geometries indicates that the current from the angular region which is not common to both geometries $\left(30^{\circ}-90^{\circ}\right.$ away from the $\theta$ direction) is more important in the wake direction than in the ram direction. The $90^{\circ}$ detector receives more current (compared with the $30^{\circ}$ detector) in the wake than it gains in the ram direction, so that the wake-to-ram current ratio is larger for the $90^{\circ}$ detector. In other words, the current gradients with respect to angle are stronger behind the satellite than in front of it. The wake gradients are examined in more detail below.

\subsection{The relative significance of $\phi_{\mathrm{N}}$ and $\mathrm{S}$ on $\alpha$-based} on Whipple's model

Figure 2 depicts graphically the parametric variation of $\alpha(120) ; \alpha(150) ; \alpha(180)$ with the normalized potential

$$
\left(\phi_{N} \equiv \frac{e \phi_{\mathrm{s}}}{K T}\right)
$$

for several values of the ion Mach number $S$ covering the range from subsonic to supersonic flows in the Whipple model for both $90^{\circ}$ and $30^{\circ}$ half-angle apertures. The conclusions derived below from this figure are valid for the parameter ranges $-3 \leqslant \phi_{\mathrm{N}} \leqslant 3$; $0.5 \leqslant S \leqslant 4.0$, i.e. applicable to plasmas which exist in the topside ionosphere and in the plasmasphere.

Some immediate conclusions are as follows.

(1) The variations of $\alpha$ with $\phi_{N}$ for constant $S$ for each angle are more pronounced for positive potential than for negative potentials in all cases.

(2) For each group of curves (i.e. for constant $S$ ), the largest change in $\alpha(\Delta \theta)$ for a unit change in $\Delta \phi_{N}$ occurs between $\phi_{\mathrm{N}}=0.0$ and $\phi_{\mathrm{N}}=+1.0$.

(3) The sensitivity of $\alpha(\Delta \theta)$ to $\phi_{N}$ is seen to increase dramatically with ion Mach number.
(4) In comparing the three curves of each model at each value of $S$, it is seen that $\alpha(120)$ is somewhat less sensitive to $\phi_{N}$ (smaller variation as $\phi_{N}$ varies from -3 to +3$)$ than are $\alpha(150)$ and $\alpha(180)$, which are roughly comparable.

(5) Ovcrall, the ratios $\alpha$ appear to depend more sensitively on the ion Mach number $S$ than on the normalized potential $\phi_{\mathrm{N}}$.

(6) For positive potentials, the $30^{\circ}$ aperture always yields a somewhat smaller normalized current than the $90^{\circ}$ aperture.

To see the fifth point more clearly, we examine the range of variations of $\alpha(180)$, first for fixed $\phi_{\mathrm{N}}$ over the range of $S$, then for fixed $S$ over the range of $\phi_{\mathrm{N}}$, using the Whipple $\left(30^{\circ}\right)$ results for maximum variation with $\phi_{\mathrm{N}}$. We do this for fixed $\phi_{\mathrm{N}}$ by taking the ratio of $\alpha(180)$ at $S=0.5$ to $\alpha(180)$ at $S=4$ for $\phi_{\mathrm{N}}=-3.0,0.0$ and +3 . For fixed $S$, we take the ratio of $\alpha(180)$ at $\phi_{\mathrm{N}}=-3$ to that at $\phi_{\mathrm{N}}=+3$ for $S=0.5$, 1.0,2.0 and 4.0. Results are presented in Table 1.

If these ranges of the parameters $S$ and $\phi_{N}$ roughly correspond to satellite observations in the plasmasphere and ionosphere, as we think they do, these results suggest that the greater variation in near-Earth observations is associated with $S$. These results also reinforce conclusion (3) that the variation with $\phi_{N}$ increases with $S$.

A significant qualitative difference between the results for the $30^{\circ}$ and $90^{\circ}$ apertures is the fact that for the $90^{\circ}$ aperture, there is no variation with negative spacecraft potential while there is for the $30^{\circ}$ aperture. While perhaps puzzling at first, the physical reason for this difference can be understood upon reffection. In the thin sheath approximation, all particles which contact the detector aperture (have some component of velocity into the detector) will enter the detector for the $90^{\circ}$ aperture acceptance case. An attractive

TABLE 1. RANGE of VARIATION OF $\alpha(180)$ BASED ON THE WHIPPLE (30 ) MODEL [EQUATION (3)]

\begin{tabular}{cccc}
\hline \multicolumn{4}{c}{ Variation with S for fixed $\phi_{N}$} \\
\\
\hline$\phi_{N}$ & -3 & 0 & +3 \\
\hline$\frac{\alpha(180 ; S=0.5)}{\alpha(180 ; S-4.0)}$ & $4.0(7)$ & $8.4(9)$ & $7.1(14)$ \\
\hline
\end{tabular}

(b) Variation with $\phi_{\mathrm{N}}$ for fixed $S$

\begin{tabular}{ccccc}
\hline$S$ & 0.5 & 1 & 2 & 4 \\
\hline$\alpha\left(180 ; \phi_{N}=-3\right)$ \\
$\alpha\left(180 ; \phi_{N}=+3\right)$ & $1.4(1)$ & $1.8(2)$ & $2.3(4)$ & $2.4(8)$ \\
\hline
\end{tabular}



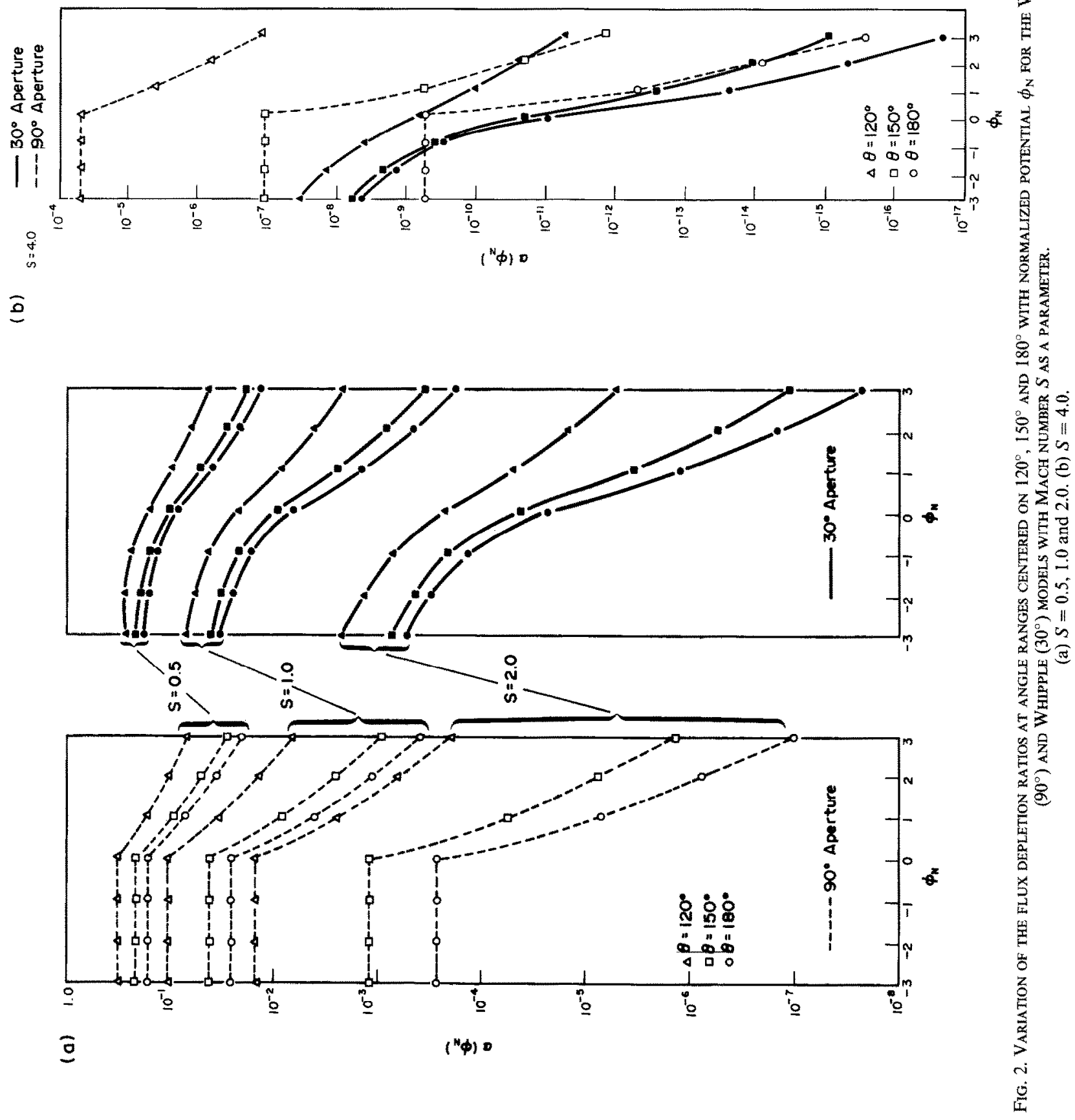
potential has no effect because of the thin sheath approximation, it cannot extend beyond the aperture plane: all the particles which can get in will get in, no matter what value the negative potential has. For the $30^{\circ}$ aperture, however, those same particles must also be within the aperture acceptance cone after passing through the thin sheath (which changes the particle direction, as discussed by Comfort et al., 1982). The effect of an attractive potential is to decrease the angle between the particle trajectory and the detector axis, thus focusing more particles into the detector. This effect increases with the magnitude of the potential.

Now it must also be recognized that what is plotted in Fig. 2 is not current into a detector but ratios of currents. For the $90^{\circ}$ aperture, which shows no variation with $\phi_{\mathrm{N}}$, this makes no difference. However, for the $30^{\circ}$ aperture, the variation of $\alpha$ with negative $\phi_{\mathrm{N}}$ will depend on whether the focusing effect is more significant in the ram direction or in the wake direction. Since $\alpha$ increases as $\phi_{\mathrm{N}}$ becomes more negative, focusing must be more important in the wake direction. This is reasonable since in the forward direction, the electrostatic potential energy would need to be comparable with the ram energy to divert (focus) the particle, whereas in the wakc it would need to bc comparable with the thermal energy. Consistent with this is the increase in the effect of the negative potential with increasing $S$.

For positive potentials similar considerations apply. In this case, the potential is repulsive for ions ; so as the potential increases, fewer particles can pass through the potential barrier. For the $30^{\circ}$ aperture in this case, a "defocusing" accurs in addition to the exclusion based solely on energy. However, this effect appears to be relatively insignificant, since even for the most sensitive case, $S=4.0$, it makes a difference of only about a factor of 3 in the variation range (about $10^{6}$ ) of $\alpha$ between $\phi_{\mathrm{N}}=0$ and $\phi_{\mathrm{N}}=3$.

\subsection{Gradients of $\alpha$ for different models}

Table 2 gives the ratios of $\alpha$ for different values of $\theta$ and $S$ in the wake region according to the Whipple, Samir and Gurevich models. As in Fig. 1, the values of $\alpha$ are averages over $\pm 15^{\circ}$ about $\theta$. Column 1 gives the value of $S$. Columns 2, 3, 4 give

$$
\left[\frac{\alpha(120)}{\alpha(180)}\right], \quad\left[\frac{\alpha(150)}{\alpha(180)}\right], \text { and }\left[\frac{\alpha(120)}{\alpha(150)}\right]
$$

according to Whipple's $\left(30^{\circ}\right)$ model $\left(\alpha_{\mathrm{W}}\right)$, evaluated for $\phi_{\mathrm{N}}=0$. Columns 5, 6, 7 and columns 8, 9, 10 give the respective values for the Samir $\left(\alpha_{S}\right)$ and the Gurevich $\left(\alpha_{\mathrm{G}}\right)$ expressions. In the Samir columns for
$S=2.0$ and 4.0 , the Whipple $\left(90^{\circ}\right)$ values are shown in brackets. The ratio

$$
\left[\frac{\alpha(120)}{\alpha(180)}\right]
$$

indicates the gradient in $\alpha$ across the entire wake region.

For all models, it is clearly seen that :

(i) the values of

$$
\left[\frac{\alpha(120)}{\alpha(180)}\right]
$$

increase significantly with increasing $S$; that is, the current vs angle profiles get steeper with increasing $S$;

(ii) the values of

$$
\left[\frac{\alpha(120)}{\alpha(150)}\right]
$$

are larger than the

$$
\left[\frac{\alpha(150)}{\alpha(180)}\right]
$$

values for all values of $S$, demonstrating that the major part of the depletion in ion current in the wake takes place in the angular range $120^{\circ} \leqslant \theta \leqslant 150^{\circ}$, which is the region of the wake closest to the undisturbed plasma. This behavior is also seen clearly in Fig. 2 for the Whipple models.

The reason for such a behavior may be connected with the region of quasineutrality where self-similar solutions hold.

It is possible that sophisticated numerical models which incorporate wave-particle interactions, turbulence, heating processes, etc., all of which are expected to take place in the wake region, may alter conclusion (ii). Although such processes are not included in the models used here, it would, nevertheless, be useful to examine conclusion (ii) with actual measurements.

\subsection{Observational conditions}

In considering the variation of $\alpha$ with Mach number and normalized potential, it should be noted that $S$ and $\phi_{\mathrm{N}}$ tend to vary systematically together over an elliptical satellite orbit, although for somewhat independent reasons. At low altitudes $(\sim 1000 \mathrm{~km})$ the spacecraft velocity is near $8 \mathrm{~km} \mathrm{~s}^{-1}$, the species is $\mathrm{O}^{+}$, densities are of the order of $10^{4} \mathrm{~cm}^{-3}$, with temperatures around $2000 \mathrm{~K}$, resulting in high ionic Mach 
TABLE 2. RATIOS OF $\alpha$ FOR DIFFERENT MODELS AND DIFFERENT VALUES OF $\theta$ AND $S$ FOR PLASMASPHERIC IONOSPHERIC CONDITIONS

\begin{tabular}{|c|c|c|c|c|c|c|c|c|c|}
\hline \multirow{3}{*}{$S$} & \multicolumn{3}{|c|}{$\alpha_{\mathrm{w}}\left(30^{\circ}\right)$} & \multicolumn{3}{|c|}{$\alpha_{\mathrm{S}}$} & \multicolumn{3}{|c|}{$\alpha_{G}$} \\
\hline & 120 & 150 & 120 & 120 & 150 & 120 & 120 & 150 & 120 \\
\hline & $\overline{180}$ & $\overline{180}$ & $\overline{150}$ & $\overline{180}$ & $\overline{180}$ & $\overline{150}$ & $\overline{180}$ & $\overline{180}$ & $\overline{150}$ \\
\hline 0.50 & 1.55 & 1.17 & 1.32 & 1.75 & 1.18 & 1.48 & 1.30 & 1.08 & 1.20 \\
\hline 1.0 & 3.18 & 1.35 & 2.36 & 4.05 & 1.53 & 2.65 & 1.94 & 1.22 & 1.59 \\
\hline 2.0 & 9.00 & 1.68 & 5.36 & $\begin{array}{c}65.0 \\
{[53.4]^{*}}\end{array}$ & $\begin{array}{c}4.24 \\
{[4.06]^{*}}\end{array}$ & $\begin{array}{c}15.3 \\
{[13.2]^{*}}\end{array}$ & 7.11 & 1.91 & 3.72 \\
\hline 4.0 & 65.2 & 2.11 & 31.0 & $\begin{array}{c}2.92(6) \\
{[9.81(4)]^{*}}\end{array}$ & $\begin{array}{c}5.89(2) \\
{[2.11(2)]^{*}}\end{array}$ & $\begin{array}{c}4.96(3) \\
{[4.66(2)]^{*}}\end{array}$ & $1.14(3)$ & 15.9 & 71.5 \\
\hline
\end{tabular}

[ ] Whipple $\left(90^{\circ}\right)$ values.

numbers $(\sim 5-6)$ and negative spacecraft potentials. On the other hand, at high altitudes, where the satellite velocities are around $3-4 \mathrm{~km} \mathrm{~s}^{-1}$, the primary ion species is $\mathrm{H}^{+}$, number densities are $\sim 10^{2} \mathrm{~cm}^{-3}$, and temperatures are around $10,000 \mathrm{~K}$, resulting in low ionic Mach numbers $(\sim 0.2)$ and positive potentials. And with potentials being normalized to $K T$, the low temperatures at low altitudes and high temperatures at high altitudes tend to result in the magnitude of $\phi_{\mathrm{N}}$ being larger for negative potentials than for positive potentials (e.g. see Paper 1).

Also, in applying the results of model calculations to actual spacecraft, it must be recognized that it is the potentials in the vicinity of the detector aperture that have the greatest influence on the particles entering the detector and ultimately being counted. This can differ substantially from the "average" spacecraft potential unless great care has been taken in the design of both the spacecraft and the detector.

A comment regarding the role of $\mathbf{H}^{+}$in explaining discrepancies associated with the neutral approximation in theory-experiment comparisons is in order. In earlier studies (e.g. Gurevich et al., 1973; Samir et al., 1981 ; Samir and Fontheim, 1981 ; Parks and Katz, 1983 ; Al'pert, 1983) it was suggested that considering relatively small concentrations of $\mathrm{H}^{+}$in the plasma may reduce theory-experiment discrepancies particularly in the low and middle ionosphere. In Samir et al. (1981), Samir and Fontheim (1981) and Parks and Katz (1983), it was suggested that theoretical models should treat separately the different ions and not use an average ionic mass and/or an average ionic Mach number. The models used in the present study use $S$ values which are based on a specific ionic mass for a single species. However, it is clear that the lower $S$ values represent the situation where $\mathrm{H}^{+}$is significant. When a mixture of ion species is observed, it is important to know the composition correctly in order to compute the wake flux correctly, within the limits of the model.

\section{SUMMARY}

We have compared several theoretical expressions which yield the angular distribution of ions around a body moving subsonically and supersonically in plasmas typical of the terrestrial plasmasphere and upper ionosphere. In these regions the $\left[\mathrm{H}^{+}\right]$ion is generally the major ionic constituent of the plasma. For the middle and lower ionosphere, $\left[\mathrm{O}^{+}\right]$is usually the major ionic constituent of the plasma.

We have shown quantitatively the degree of variability among the different models (expressions) and showed quantitatively the relative significance of the ionic Mach number $(S)$ and the normalized body potential $\left(\phi_{\mathrm{N}}\right)$ in determining the current ratio

$$
\alpha=\frac{I_{+}(\theta)}{I_{+}(\mathrm{ram})}
$$

over the angular $(\theta)$ range in the wake. In the ranges $0.5 \leqslant S \leqslant 4.0$ and $-3.0 \leqslant \phi_{\mathrm{N}} \leqslant+3.0$, we find the ionic Mach number to be more significant in determining $\alpha$. Furthermore, the relative significance of $\phi_{N}$ increases with $S$.

Substantial effects are found when the detector aperture is limited in angular acceptance, as is found in several recent spacecraft instruments. The effect is generally to decrease the wake depletion ratio by an amount which increases with increasing Mach number. Limited apertures are also found to affect the response to body potentials, with significant "focusing" occurring for attractive potentials, which have no effect on detectors with full hemispheric acceptance. 
Another result of interest is that according to the simple models examined here, the main depletion in ion current in the wake region takes place in the angular range of $120^{\circ} \leqslant \theta \leqslant 150^{\circ}$. This may be related to the region of quasi-neutrality which exists in the wake "shoulders", where, in terms of "plasma expansion", self-similar solutions hold (e.g. Samir et al., 1983; Singh and Schunk, 1982; Gurevich et al., 1969, 1973). This is the region closest to the plasma-vacuum interface.

Combining theoretical conclusions from the present study with theoretical and experimental (in situ) results from earlier investigations, e.g. Henderson and Samir (1967), Samir and Wrenn (1969), Gurevich et al. (1969, 1973), Samir and Stone (1980), Stone (1981a,b), Al'pert (1983), Samir et al. (1986), we suggest the following.

(1) The neutral approximation, despite its shortcomings, is useful for some conditions pertaining in the plasmasphere, and in the upper ionosphere. Neutral approximation models are more applicable to plasmas where the $\left[\mathrm{H}^{+}\right]$ion is the dominant ionic constituent of the plasma.

(2) Earlier studies (e.g. Samir et al., 1973; Samir and Fontheim, 1981; and others) have shown that simple neutral approximations are not applicable in theory-expcriment comparisons for conditions pertaining to the lower ionosphere. This is particularly true in the "maximum rarefaction region", i.e. for angles of attack larger than approximately $120^{\circ}-130^{\circ}$ in the vicinity of the spacecraft surface. However, for regions downstream from the body surface and/or for angular regions distant from the wake axis, a neutral approximation may still be valid for specific cases (e.g. Gurevich et al., 1969, 1973; Al'pert, 1983).

It is expected that the above suggestions will be useful in body-plasma interaction research in the plasmasphere. It should be noted that the present study has not addressed large objects, such as the shuttle/ space station. Moreover, for certain conditions, the applicability can be studied in the laboratory, where control over a variety of body and plasma parameters can be maintained.

Acknowledgements-The computer program used to evaluate the Whipple expression was modified from one originally written by N. Singh. U.S. acknowledges the support of the NASA grant NGR-23-005-320 and the interest of S. D. Shawhan at NASA Headquarters. R.H.C. and K.H.W. were partially supported by NASA contract NAS8-33982 and NASA grant NAG8-058 with The University of Alabama in Huntsville. Finally, we appreciate the constructive comments made by the referee.

\section{REFERENCES}

Al'pert, Y. L. (1983) The Near-Earth and Interplanetary Plasma, Vol. 2, Plasma Flow, Plasma Waves, and Oscillations. Cambridge University Press, New York.

Comfort, R. H., Baugher, C. R. and Chappell, C. R. (1982) Use of the thin sheath approximation for obtaining ion temperatures from the ISEE-1 limited aperture RPA. $J$. geophys. Res. 87, 5109.

Comfort, R. H., Waite, J. H., Jr. and Chappell, C. R. (1985) Thermal ion temperatures from the retarding ion mass spectrometer on $D E-1 . J$.geophys. Res. 90, 3475 .

Gurevich, A. V. and Meshcherkin, A. P. (1981a) Ion acceleration in an expanding plasma. Sm. Phys. IETP (Fngl. Trans.) 53, 937.

Gurevich, A. V. and Meshcherkin, A. P. (1981b) Jump discontinuity on the front of a rarefaction wave in a plasma. Sov. Phys. JETP (Engl. Trans.) 54, 688.

Gurevich, A. V., Pariskaya, L. V. and Pitaevsky, L. P. (1973) Ion acceleration upon expansion of a rarefied plasma. Sov. Phys. IETP (Engl. Trans.) 36, 274.

Gurevich, A. V. and Pitaevsky, L. P. (1975) Nonlinear dynamics of a rarefied ionized gas. Prog. Aerospace Sci. 16, 227.

Gurevich, A. V., Pitaevsky, L. P. and Smimova, V: V. (1969) Ionospheric aerodynamics. Space Sci. Rev. 9, 805 .

Henderson, C. L. and Samir, U. (1967) Observations of the disturbed region around an ionospheric spacecraft. Planet. Space Sci. 15, 1499.

Katz, I., Cooke, D. L., Parks, D. E. and Mandell, M. J. (1984) Three dimensional wake model for low earth orbit. J. Spacecraft Rockets 21, 125.

Murphy, G. B., Pickett, J., D'Angelo, N. and Kurth, W. S. (1986) Measurements of plasma parameters in the vicinity of the space shuttle. Planet. Space Sci. 34, 993.

Parker, L. W. (1977) Calculation of sheath and wake structure about a pillbox-shaped spacecraft in a flowing plasma. Proc. Spacecraft Charging Technology Conference (Edited by Pike, C. P. and Lowell, R. R.), AFGL-TR-77-005/ NASA TMX-73537, pp, 331-366.

Parker, L. W. (1983) Contributions to satellite sheath and wake modeling. Proc. Spacecraft/Plasma Interactions, 17th ESLAB Symp. (Edited by Pedersen, A., Guyenne, D. and Hunt, J.), ESA SP-198, pp. 81-101.

Parks, D. E. and Katz, I. (1983) Mechanisms that limit potentials on ionospheric satellite. $J$. geophys. Res. 88, 9155.

Raitt, W. J., Siskind, D. E., Banks, P. M. and Williamson, P. R. (1984) Measurements of the thermal plasma environment of the space shuttle. Planet. Space Sci. 32, 457.

Samir, U., Comfort, R. H., Chappell, C. R. and Stone, N. H. (1986) Observations of low-energy ions in the wake of a magnetospheric satellite. $J$. geophys. Res. 91, 5725 .

Samir, U. and Fontheim, E. G. (1981) Comparison of theory and in situ observations for electron and ion distributions in the near wake of Explorer 31 and Atmosphere Explorer C satellites. Planet. Space Sci. 29, 975.

Samir, U., Maier, E. J. and Troy, B. E., Jr. (1973) The angular distribution of ion fux around an ionospheric satellite. $J$. atmos, terr. Phys. 35,53 .

Samir, U. and Stone, N. H. (1980) Shuttle-era experiments in the area of plasma flow interactions with bodies in space. Acta Astronautica 7, 1091.

Samir, U. and Widjaja, D. (1980) On the significance of including the thermal motion of ions in determining the ion distribution behind a satellite. $J$. geophys. Res. 86, 4807 . 
Samir, U., Wildman, P., Rich, F., Brinton, H. and Sagalyn, R. C. (1981) About the parametric interplay between ionic Mach number, body Si3e and satellite potential in determining the ion depletion in the wake of the $\mathbf{3} 3-2$ satellite. J. geophys. Res. 86, 11161.

Samir, U. and Willmore, A. P. (1965) The distribution of charged particles near a moving spacecraft. Planet. Spoce Sci. 13,285 .

Samir, U. and Wrenn, G. L. (1969) The dependence of charge and potential distribution around a spacecraft on ionic composition. Planet. Space Sci. 17, 693.

Samir, U., Wright, K. H., Jr. and Stone, N. H. (1983) The expansion of a plasma into a vacuum: basic phenomena and processes and applications into space plasma physics. Rev. Geophys. 21, 1631.

Singh, N. and Schunk, R. W. (1982) Numerical calculations relevant to the initial expansion of the polar wind. $J$. geophys. Res. 87, 9154.

Siskind, D. E., Raitt, W. J., Banks, P. M. and Williamson, P. R. (1984) Interactions between the orbiting space shuttle and the ionosphere. Planet. Space Sci. 32, 881 .
Stone, N. H. (1981a) The aerodynamics of bodies in a rarefied ionized gas with applications to spacecraft environmental dynamics. NASA Tech. Paper 1933, Washington, DC.

Stone, N. H. (1981b) The plasma wake of mesosonic conducting bodies. Part 1. An experimental parametric study of ions focusing by the plasma sheath. J. plasma Phys. 25, 351.

Whipple, E. C. (1959) The ion-trap results in exploration of the upper atmosphere with the help of the third Soviet Sputnik. Proc. IRE 47, 2023.

Whipple, E. C., Warnock, J. M. and Winkler, R. H. (1974) Effect of a satellite potential on direct density measurements through the plasmapause. J. geophys. Res. 79, 179.

Wright, K. H., Jr., Parks, D. E., Katz, I, Stone, N. H. and Samir, U. (1986) More on the expansion of a collisionless plasma into the wake of a body. J. plasma Phys. 35, 119.

Wright, K. H., Jr., Stone, N. H. and Samir, U. (1985) A study of plasma expansion phenomena in laboratory generated plasma wakes : preliminary results. J. plasma Phys. 33, 71. 